\title{
Gestational weight management and pregnancy outcomes among women of advanced maternal age
}

\author{
JUAN LIN*, YUELIN FU*, QING HAN, JIANYING YAN, RONGXIN CHEN and HUALE ZHANG \\ Department of Obstetrics, Fujian Provincial Maternity and Children's Hospital, Affiliated \\ Hospital of Fujian Medical University, Fuzhou, Fujian 350001, P.R. China
}

Received May 14, 2019; Accepted July 3, 2019

DOI: $10.3892 /$ etm.2019.7752

\begin{abstract}
In this study, we assessed the effects of pre-pregnancy body mass index (BMI) and gestational weight gain (GWG) on the pregnancy outcomes of women of advanced age using a back-propagation (BP) artificial neural network. We conducted a retrospective analysis on postpartum and hospital delivery data from 1,015 women of advanced maternal age (AMA) hospitalized at the Fujian Provincial Maternity and Children's Hospital from January to June, 2017. Pre-pregnancy overweight was found to increase the incidence of gestational diabetes mellitus (GDM), hypertensive disorders complicating pregnancy (HDCP) and fetal macrosomia. In addition, poor weight gain during pregnancy increased the chances of pre-term births (PTBs). Furthermore, excessive weight gain during pregnancy increased the incidence of macrosomia in women of AMA. On the whole, the findings of this study suggest that controlling the pre-pregnancy BMI and the GWG may reduce the incidence of adverse pregnancy outcomes in women of AMA. The BP neural network is suitable for the study of weight changes in this population.
\end{abstract}

\section{Introduction}

Pregnancy weight management is a goal of prenatal care in China. Since the implementation of the two-child policy, the proportion of pregnant women of advanced maternal age (AMA, pregnant women $>35$ years of age) has increased; this proportion accounts for approximately $60 \%$ of all pregnancies, and half of these women are older than 40 years (1). Women

Correspondence to: Dr Jianying Yan, Department of Obstetrics, Fujian Provincial Maternity and Children's Hospital, Affiliated Hospital of Fujian Medical University, 18 Daoshan Road, Gulou, Fuzhou, Fujian 350001, P.R. China

E-mail: yanjy2004@yeah.net

*Contributed equally

Key words: advanced maternal age, pre-pregnancy body mass index, gestational weight gain, pregnancy outcome of AMA are more frequently overweight before the pregnancy (2), and are more likely to have had internal and surgical diseases, and are more likely to be multiparas; in addition, age is an independent risk factor for maternal and child adverse outcomes (3). Therefore, AMA pregnancy management aims to improve pregnancy outcomes.

Pre-pregnancy overweight may lead to gestational diabetes mellitus (GDM), hypertensive disorders complicating pregnancy (HDCP), fetal macrosomia, and other adverse pregnancy outcomes (4). Women of AMA may disregard pregnancy weight management recommendations, and excessive weight gain during pregnancy can increase the incidence of adverse outcomes (5).

Controlling pre-pregnancy weight and gestational weight gain $(\mathrm{GWG})$ reduces the incidence of GDM, HDCP, preterm births (PTBs), shoulder dystocia and the incidence of large for gestational age (LGA) births; weight control increases vaginal delivery rates, and decreases the incidence of low birth weight and of small for gestational age (SGA) births (6). Therefore, the evidence-based management of pre-pregnancy and gestational weights maximizes good pregnancy outcomes.

In this study, we analyzed the clinical data of 1,015 women of AMA admitted to Fujian Provincial Maternity and Children's Hospital to detect associations between their pre-pregnancy body mass indexes (BMIs) or GWGs and their pregnancy outcomes.

\section{Materials and methods}

Study population. From January to June, 2017, 1,015 women of AMA delivered babies at the Fujian Provincial Maternity and Children's Hospital. We included data from women older than 35 years of age at delivery with single pregnancies who had had $>5$ prenatal checkups We excluded data from women with internal and surgical diseases, and those with incomplete information. The Ethics Committee of the Fujian Provincial Maternity and Children's Hospital approved the study and waived the need for informed consents given its retrospective nature.

Research methods. We collected medical and hospitalization information, including data on pregnancy outcomes (GDM, HDCP and PTB) and neonatal complications (macrosomia and SGA). Data were grouped according to the women's age 
during childbirth into an advanced age group (35-40 years) and a super-advanced age group ( $\geq 40$ years).

Alternatively, data were grouped according to the women's pre-pregnancy BMIs [according to the guidelines for pregnancy weight management published by the National Institute of Medicine (IOM) in 2009 (7)] into the underweight group (BMI $<18.5 \mathrm{~kg} / \mathrm{m}^{2}$ ) with 105 cases; normal weight (BMI 18.5-24.9 kg/m²) group with 789 cases; overweight group (BMI 25.0-29.9 kg/m²) with 107 cases; and, obese group (BMI $\geq 30 \mathrm{~kg} / \mathrm{m}^{2}$ ) with 14 cases. Finally, we also subdivided data according to GWG among women with normal BMIs (IOM guidelines) into 3 subgroups as follows: A (GWG $<11.5 \mathrm{~kg}$ with 188 cases), B (GWG between 11.5 and $16.0 \mathrm{~kg}$ with 405 cases), and C (GWG $>16.0 \mathrm{~kg}$ with 196 cases). We compared the mean variables and outcome incidences between the groups.

Statistical analysis. We performed all statistical analyses using SPSS 21.0 software (SPSS, Inc.). We calculated measurement data as the means \pm standard deviation (SD). We used one-way ANOVA for testing hypotheses between groups and used the Scheffe test for comparing between groups to prove the significance of ANOVA. Chi-square tests for qualitative difference analyses. We applied a logistic regression analysis to examine the influence of variables on the pregnancy outcomes. Values of $\mathrm{P}<0.05$ were considered to indicate statistically significant differences.

Back-propagation (BP) neural network applications. Among the 1,015 women, 800 cases of AMA were randomly selected as the training dataset. The BP neural network is a supervised model divided into 3 layers (input, hidden and output) (8). In this study, we designed a 3-layer BP neural network to examine the effects of pre-pregnancy BMI and GWG on pregnancy outcomes. We set the characteristic value $\mathrm{x} 1$ as the pre-pregnancy BMI and $\mathrm{x} 2$ as the GWG; the output value $\mathrm{y} 1$ included GDM, HDCP, PTB, macrosomia, SGA and neonatal birth weight (NBW). The two input values (x1 and $\mathrm{x} 2$ ) were set as the input layer nodes, and the output values were set as the output layer nodes (y1) (Fig. S1).

\section{Results}

General conditions of women of AMA. We collected clinical data from 1,015 women, 809 in the advanced-age group (79.7\%) and 206 in the super-advanced age group (20.3\%). Among the women in the advanced age group, 93 (11.50\%) were underweight, $623(77.01 \%)$ were at a normal weight, $83(10.26 \%)$ were overweight and $10(1.23 \%)$ were obese. In addition, among the women in the super-advanced age group, $12(5.83 \%)$ were underweight, $166(80.58 \%)$ were at a normal weight, 24 (11.65\%) were overweight and 4 (1.94\%) were obese. The women in the super advanced maternal age group had a higher incidence of overweight and obesity than those in the advanced maternal age group $(\mathrm{P}<0.05)$ (Table I).

Association between maternal age at birth and pregnancy outcome. We found a higher incidence of GDM, HDCP and macrosomia in the super-advanced maternal age group than in the advanced age group $(\mathrm{P}<0.05$; Table II).
Association between pre-pregnancy BMI and pregnancy outcome. As shown in Table III, overweight women had a significantly greater incidences of GDM, HDCP and fetal macrosomia than women who were at a normal weight (both $\mathrm{P}<0.05)$. In addition, birth weight differed significantly among the 4 groups $(\mathrm{P}<0.05)$.

Association between $G W G$ and pregnancy outcome in the normal BMI group. The incidence of GDM in the low GWG group (group A) was $38.30 \%$, which was higher than that in the normal GWG group (group B; 27.41\%). The risk of developing HDCP and macrosomia was also increased in women with a high GWG. The risks of PTB were higher in women with low GWG than those in the normal GWG group (group B). The mean NBWs differed between the 3 groups of mothers (Table III).

BP neural network training results. Data from 800 women of AMA were randomly selected for the training dataset. We used the pre-pregnancy BMI as input $\mathrm{x} 1$, the GWG as input $\mathrm{x} 2$, and the pregnancy outcome as output $\mathrm{y} 1$. We factored in 6 pregnancy outcomes (GDM, HDCP, PTB, macrosomia, SGA and NBW). As a result, we established 6 neural networks to train the 6 pregnancy outcomes separately. We used data from 215 women of AMA and pre-pregnancy BMI, GWG and pregnancy outcomes to validate the BP neural network training model. The prediction accuracy of the 6 pregnancy outcomes from the BP neural network training model is presented in Table IV.

We compared the weighted influence of pre-pregnancy BMI and GWG on the pregnancy outcomes. Pre-pregnancy BMI affects the development of GDM by about half the influence of GWG; the pre-pregnancy BMI and GWG had similar influences on HDCP and macrosomia. The influence of pre-pregnancy BMI on SGA and on NBW was slightly greater than that of GWG on the same outcomes (Table IV).

\section{Discussion}

Women of AMA are more likely to be overweight and this factor, together with other medical variables, increases their risk of maternal and child adverse outcomes $(1,3)$. Prenatal education and weight control can improve pregnancy outcomes.

Association of pre-pregnancy weight and pregnancy outcomes for women with AMA. Age is a risk factor for pre-pregnancy overweight (2), and overweight can trigger GDM, HDCP, macrosomia and other adverse pregnancy outcomes (9-11). In this study, we demonstrated that women of AMA and overweight were more likely to develop GDM than women with normal pre-pregnancy BMIs. A previous study demonstrated that the risk of developing GDM among women who were overweight before pregnancy was greater than the risk among pregnant women with normal pre-pregnancy weights (2). The body's glucose tolerance becomes impaired with age due to gradual islet $\beta$-cell dysfunction (12), and being overweight is associated with insulin resistance, insulin secretion abnormalities, and insulin receptor abnormalities(1). Thus, women of AMA and who are overweight are more prone to GDM during pregnancy than their younger counterparts. The prevalence of HDCP in obese pregnant women is up to 6-fold greater than 
Table I. Maternal baseline characteristics between women in the advanced-age group and those in the super-advanced age group.

\begin{tabular}{|c|c|c|c|c|}
\hline Characteristic & $\begin{array}{l}\text { Advanced age } \\
\text { group n }(\%)\end{array}$ & $\begin{array}{c}\text { Super-advanced age } \\
\text { group n }(\%)\end{array}$ & $\chi^{2}$ & P-value \\
\hline Total population & 809 (100) & $206(100)$ & - & - \\
\hline \multicolumn{5}{|l|}{ Educational attainment } \\
\hline Bachelor degree or below & $185(22.87)$ & $46(22.33)$ & 0.03 & 0.87 \\
\hline Bachelor degree or above & $624(77.13)$ & $160(77.67)$ & & \\
\hline \multicolumn{5}{|l|}{ Parity (times) } \\
\hline$<2$ & $764(94.44)$ & $195(94.66)$ & 0.02 & 0.9 \\
\hline$\geq 2$ & $45(5.56)$ & $11(5.34)$ & & \\
\hline History of cesarean section & $323(39.93)$ & $83(40.29)$ & 0.01 & 0.92 \\
\hline \multicolumn{5}{|l|}{ Pre-pregnancy BMI category } \\
\hline Underweight & $93(11.50)$ & $12(5.83)$ & 4.54 & $0.03^{\mathrm{a}}$ \\
\hline Normal weight & $623(77.01)$ & $166(80.58)$ & & \\
\hline Overweight & $83(10.26)$ & $24(11.65)$ & & \\
\hline Obese & $10(1.23)$ & $4(1.94)$ & & \\
\hline
\end{tabular}

andicates statistical significance $(\mathrm{P}<0.05)$.

Table II. Comparison of pregnancy outcomes between women of advanced age and those with a super-advanced age.

\begin{tabular}{|c|c|c|c|c|}
\hline & $\begin{array}{l}\text { Advanced age group } \\
\quad(\mathrm{n}=809), \mathrm{n}(\%)\end{array}$ & $\begin{array}{l}\text { Super-advanced age group no } \\
\qquad(\mathrm{n}=206), \mathrm{n}(\%)\end{array}$ & $\chi^{2}$ & P-value \\
\hline \multicolumn{5}{|c|}{ GDM } \\
\hline $\mathrm{Y}$ & $229(28.31)$ & 78 (37.86) & 7.11 & $0.01^{\mathrm{b}}$ \\
\hline $\mathrm{N}$ & $580(71.69)$ & $128(62.14)$ & & \\
\hline \multicolumn{5}{|c|}{ HDCP } \\
\hline Y & 25 (3.09) & $11(5.34)$ & 2.43 & $0.02^{\mathrm{a}}$ \\
\hline $\mathrm{N}$ & 784 (96.91) & 195 (94.66) & & \\
\hline \multicolumn{5}{|c|}{ РTB } \\
\hline $\mathrm{Y}$ & $29(3.58)$ & $10(4.85)$ & 0.72 & 0.4 \\
\hline $\mathrm{N}$ & $780(96.42)$ & $196(95.15)$ & & \\
\hline \multicolumn{5}{|c|}{ Macrosomia } \\
\hline $\mathrm{Y}$ & $56(6.92)$ & $19(9.22)$ & 0.4 & $0.03^{\mathrm{a}}$ \\
\hline $\mathrm{N}$ & $753(93.08)$ & $187(90.78)$ & & \\
\hline \multicolumn{5}{|c|}{ SGA } \\
\hline $\mathrm{Y}$ & $15(1.85)$ & $3(1.46)$ & 0.15 & 0.7 \\
\hline $\mathrm{N}$ & 794 (98.15) & 203 (98.54) & & \\
\hline
\end{tabular}

${ }^{\mathrm{a}} \mathrm{P}<0.05$ and ${ }^{\mathrm{b}} \mathrm{P} \leq 0.01$. Y represents women with this symptom, $\mathrm{N}$ represents women without this symptom. Comparisons were only made between the women with symptoms group in the advanced age group and super-advance age group. GDM, gestational diabetes mellitus; HDCP, hypertensive disorder complicating pregnancy; PTB, pre-term birth; SGA, small gestational age.

that in pregnant women with a normal weight (10), and the probability of developing preeclampsia is also higher among these women (13). A multicenter cohort study on the association between pre-pregnancy BMI and pregnancy outcomes found that the incidence of pre-eclampsia increased with maternal pre-pregnancy weight (14). Women of AMA undergo progressive damage to their uterine myometrial vascular endothelial cells (decreased vasoconstriction and sclerosis), which causes ischemia or oxidative stress. In addition, the adipose tissue in women of AMA who are also overweight secretes excessive adipokines that increase blood pressure during pregnancy. The results of this study demonstrated that women of AMA and high pre-pregnancy BMIs had the greatest risk of developing HDCP.

The occurrence of PTB and pre-pregnancy BMI in this study had a 'U'-shaped association, with the incidence of PTB 


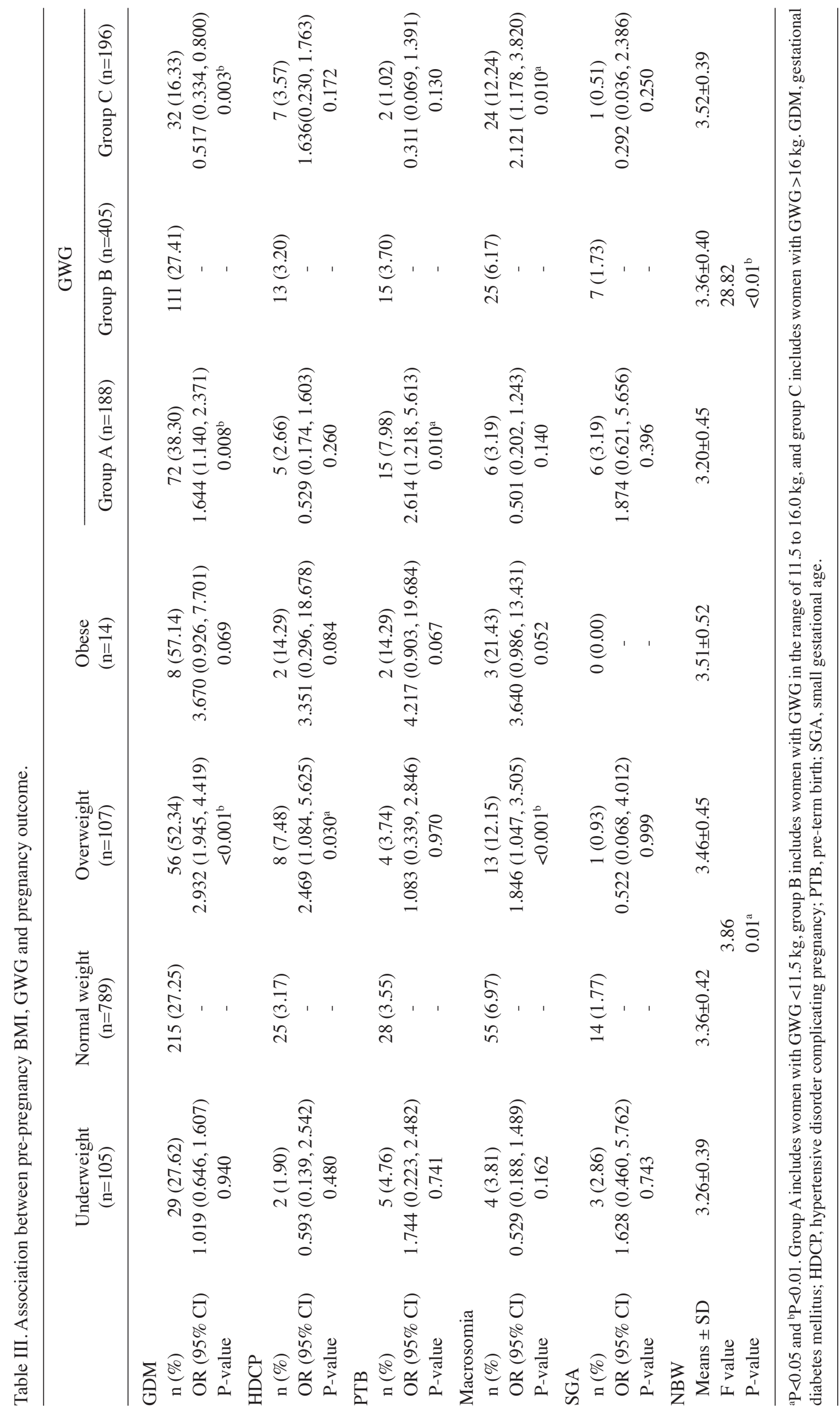


Table IV. BP network predicts the accuracy of 6 pregnancy outcomes and the importance of pre-pregnancy BMI and GWG for pregnancy outcomes.

\begin{tabular}{lccc}
\hline $\begin{array}{l}\text { Pregnancy } \\
\text { outcomes }\end{array}$ & $\begin{array}{c}\text { BP } \\
\text { Accuracy }\end{array}$ & $\begin{array}{c}\text { Pre-pregnancy } \\
\text { weight }\end{array}$ & GWG \\
\hline GDM & $89 \%$ & 0.68 & 1.31 \\
HDCP & $91 \%$ & 1.16 & 1.15 \\
PTB & $90 \%$ & 0.57 & 1.28 \\
Macrosomia & $85 \%$ & 1.22 & 1.23 \\
SGA & $85 \%$ & 1.35 & 1.17 \\
NBW & $86 \%$ & 2.26 & 1.95 \\
\hline
\end{tabular}

$\mathrm{BP}$ accuracy presents the result of 6 pregnancy outcomes prediction by BP network. BP, back-propagation' GDM, gestational diabetes mellitus; HDCP, hypertensive disorder complicating pregnancy; PTB, pre-term birth; SGA, small gestational age.

being higher in women with low pre-pregnancy BMIs, lower in women with normal BMIs, and higher again in women with high pre-pregnancy BMIs. This may be explained by nutrient deficiencies with insufficient body fluid expansion in women with low BMIs that increases the spontaneous incidence of PTB (15), and by the triggering of gestational hypertension, pre-eclampsia and other inadvertent pregnancy outcomes that can lead to an increase in iatrogenic PTB in women with pre-pregnancy overweight (16). Pre-pregnancy BMIs and GWGs have been shown to be positively associated with NBW and macrosomia, and to be negatively associated with SGA (4).

A meta-analysis of global pregnancy cases demonstrated that a low BMI increased the risk of SGA, while a high BMI decreased this risk (17). Women of AMA tend to present with hyperlipidemia (1), and higher GWGs than younger women. In addition, women of AMA are more often diabetic, and more prone to developing GDM, which increases the chance of macrosomia $(9,18)$. The incidence of macrosomia in this study increased with the BMIs of the mothers. In addition, the risk SGA was greater in women with low pre-pregnancy BMIs than in women with normal pre-pregnancy BMIs; however, women with high pre-pregnancy BMI had a decreased risk of SGA.

Association between $G W G$ and pregnancy outcomes in women of AMA. We found low GWG to be a risk factor for GDM. The results of BP neural network revealed that the effect of GWG on GDM was 2-fold stronger than that of pre-pregnancy BMI, similarly to the results of a previous study (9). In this study, the women who had regular prenatal checkups received dietary and exercise guidance after receiving a diagnosis of GDM. Therefore, they conducted routine blood glucose monitoring and controlled their weight for the rest of the pregnancy. Strict pregnancy weight management should maintain a lower GWG for women with GDM than for women without GDM (4). The results of this study revealed that the risk of HDCP in women with excessive GWG was greater than that in women with normal GWG, but insufficient GWG was not associated with HDCP. Although excessive GWG is positively associated with HDCP, distinguishing the cause and effect between GWG and HDCP is difficult. Excessive weight gain may increase the incidence of HDCP; however, HDCP induces edema and fluid retention, thus leading to increased weight (10).

The association between GWG and PTB is also controversial. In this study, we found that the incidence of PTB in women with insufficient GWG was greater than that in women with normal GWG. The results of our BP neural network revealed that the influence of GWG on the risk of PTB was 2-fold greater than the influence of pre-pregnancy BMI, suggesting that insufficient GWG is the main cause of PTB. In addition, the risk of macrosomia in women with excessive GWG was greater than that in women with normal GWG. Insufficient GWG was not associated with macrosomia. A previous study found a greater risk of macrosomia in women with excessive GWG than that in women with normal GWG (19). In this study, we did not observe reductions in the risk of macrosomia in women with insufficient GWG. In addition, the risk of SGA in women with insufficient GWG was greater than that in women with normal GWG, and excessive GWG was not associated with SGA. Studies have shown indirect associations between SGA and GWG $(10,20)$.

In conclusion, according to the findings of this study, among women of AMA, compared with with those who are at a normal weight pre-pregnancy, being overweight increased the risk of GDM, HDCP and macrosomia.The incidence of macrosomia was higher in the group of women who gained more weight during pregnancy than those who met the IOM standard. On the contrary, the incidence of PTB was higher in the group of women who gained less weight during pregnancy than in the group whose GWG met the IOM standard. According to the recommended IOM guidelines. Women of AMA should control their pre-pregnancy BMI and maintain a normal GWG to diminish the risks of adverse pregnancy outcomes. The BP neural network is a suitable approach for the study of weight changes in women with AMA during pregnancy.

\section{Acknowledgements}

Not applicable.

\section{Funding}

This study was supported by the Fujian Science and Technology Key Project (2018Y0005), and Fujian Medical Innovation Subject (2017-CX-11), and the Key Clinical Specialty Discipline Construction of Fujian, P.R. China [(2015) no. 593].

\section{Availability of data and materials}

The datasets used and/or analyzed during the current study are available from the corresponding author on reasonable request.

\section{Authors' contributions}

JL, YF and JY conceived and designed the study; QH, RC and $\mathrm{HZ}$ collected and analyzed the data of this study; JL, YF and $\mathrm{QH}$ wrote the manuscript; and JY reviewed and edited the manuscript. All authors have read and approved the final manuscript. 


\section{Ethics approval and consent to participate}

The Ethics Committee of the Fujian Provincial Maternity and Children's Hospital approved this retrospective medical record review (approval no. 2017-CX-11).

\section{Patient consent for publication}

Not applicable.

\section{Competing interests}

The authors declare that they have no competing interests.

\section{References}

1. Chen Y,Zheng XL, Wu SW and Zhang WY: Clinic characteristics of women with advanced maternal age and perinatal outcomes. Zhonghua Fu Chan Ke Za Zhi 52: 508-513, 2017 (In Chinese).

2. Dong B, Yu H, Wei Q, Zhi M, Wu C, Zhu X and Li L: The effect of pre-pregnancy body mass index and excessive gestational weight gain on the risk of gestational diabetes in advanced maternal age. Oncotarget 8: 58364-58371, 2017.

3. Wang C, Wang XY and Yang HX: Effect of maternal age on pregnancy outcomes in Beijing. Zhonghua $\mathrm{Fu}$ Chan $\mathrm{Ke} \mathrm{Za}$ Zhi 52: 514-520, 2017.

4. Yang S, Peng A, Wei S, Wu J, Zhao J, Zhang Y, Wang J, Lu Y, Yu Y and Zhang B: Pre-Pregnancy Body Mass Index, Gestational Weight Gain, and Birth Weight: A Cohort Study in China. PLoS One 10: e0130101, 2015.

5. Hill B, McPhie S, Moran LJ, Harrison P, Huang TT-K, Teede H and Skouteris $\mathrm{H}$ : Lifestyle intervention to prevent obesity during pregnancy: Implications and recommendations for research and implementation. Midwifery 49: 13-18, 2017.

6. Di Mascio D, Magro-Malosso ER, Saccone G, Marhefka GD and Berghella V: Exercise during pregnancy in normal-weight women and risk of preterm birth: a systematic review and meta-analysis of randomized controlled trials. Am J Obstet Gynecol 215: 561-571, 2016.

7. Institute of Medicine (US) and National Research Council (US) Committee to Reexamine IOM Pregnancy Weight Guidelines; Rasmussen KM and Yaktine AL (eds): Weight Gain During Pregnancy: Reexamining the Guidelines. National Academies Press, Washington, DC, 2009.

8. Jin D and Lin S (eds): Brief introduction of back propagation (BP) neural network algorithm and its improvement. In: Advances in Computer Science and Information Engineering. Springer, Berlin, Heidelberg, pp553-558, 2012.
9. Yang S, Zhou A, Xiong C, Yang R, Bassig BA, Hu R, Zhang Y, Yao C, Zhang Y, Qiu L, et al: Parental body mass index, gestational weight gain, and risk of macrosomia: A population-based case-control study in China. Paediatr Perinat Epidemiol 29: 462-471, 2015.

10. Bautista-Castaño I, Henriquez-Sanchez P, Alemán-Perez N, Garcia-Salvador JJ, Gonzalez-Quesada A, García-Hernández JA and Serra-Majem L: Maternal obesity in early pregnancy and risk of adverse outcomes. PLoS One 8: e80410, 2013.

11. Liu L, Hong Z and Zhang L: Associations of prepregnancy body mass index and gestational weight gain with pregnancy outcomes in nulliparous women delivering single live babies. Sci Rep 5: $12863,2015$.

12. Helseth R, Salvesen O, Stafne SN, Mørkved S, Salvesen KA and Carlsen SM: Gestational diabetes mellitus among Nordic Caucasian women: Prevalence and risk factors according to WHO and simplified IADPSG criteria. Scand J Clin Lab Invest 74: 620-628, 2014.

13. Roberts JM, Bodnar LM, Patrick TE and Powers RW: The role of obesity in $\pi$ reeclampsia. Pregnancy Hypertens 1: 6-16, 2011.

14. Schummers L, Hutcheon JA, Bodnar LM, Lieberman E and Himes KP: Risk of adverse pregnancy outcomes by prepregnancy body mass index: A population-based study to inform prepregnancy weight loss counseling. Obstet Gynecol 125: 133-143, 2015.

15. Huang A, Ji Z, Zhao W, Hu H, Yang Q and Chen D: Rate of gestational weight gain and preterm birth in relation to prepregnancy body mass indices and trimester: A follow-up study in China. Reprod Health 13: 93, 2016.

16. Drehmer M, Duncan BB, Kac G and Schmidt MI: Association of second and third trimester weight gain in pregnancy with maternal and fetal outcomes. PLoS One 8: e54704, 2013.

17. Liu P, Xu L, Wang Y,Zhang Y,Du Y, Sun Y and Wang Z: Association between perinatal outcomes and maternal pre-pregnancy body mass index. Obes Rev 17: 1091-1102, 2016.

18. Wang S, Ge X, Zhu B, Xuan Y, Huang K, Rutayisire E, Mao L, Huang S, Yan S and Tao F: Maternal continuing folic acid supplementation after the first trimester of pregnancy increased the risk of large-for-gestational-age birth: A Population-Based Birth Cohort Study. Nutrients 8: 8, 2016.

19. Gaillard R, Durmus B, Hofman A, Mackenbach JP, Steegers EA and Jaddoe VW: Risk factors and outcomes of maternal obesity and excessive weight gain during pregnancy. Obesity (Silver Spring) 21: 1046-1055, 2013.

20. Goldstein RF, Abell SK, Ranasinha S, Misso M, Boyle JA, Black MH, Li N, Hu G, Corrado F, Rode L, et al: Association of gestational weight gain with maternal and infant outcomes: A systematic review and meta-analysis. JAMA317: 2207-2225, 2017. 